\title{
RhoA inhibits apoptosis and increases proliferation of cultured SPCA1 lung cancer cells
}

\author{
DABEI LIU ${ }^{1,2}$, XINGKE MEI $^{1,2}$, LINLIN WANG ${ }^{1,2}$ and XUEYING YANG ${ }^{1}$ \\ ${ }^{1}$ Department of Thoracic Surgery, The Fourth Affiliated Hospital of China Medical University, Shenyang, Liaoning 110032; \\ ${ }^{2}$ Department of Thoracic Surgery, Shengjing Hospital of China Medical University, Shenyang, Liaoning 110004, P.R. China
}

Received January 20, 2016; Accepted January 18, 2017

DOI: $10.3892 / \mathrm{mmr} .2017 .6545$

\begin{abstract}
The Rho kinase pathway has previously been reported to possess a close relationship with the growth, migration and invasion of lung cancer cells. However, the molecular mechanisms underlying the effects of this pathway on lung cancer cells are still elusive. The aim of the present study was to investigate the effects and underlying molecular mechanisms of Ras homolog family member A (RhoA) on the proliferation and apoptosis of SPCA1 lung carcinoma cells. Stable SPCA1 lung cancer cell lines, in which RhoA expression was silenced by small interfering RNA, were isolated following Geneticin screening. Inhibition of RhoA expression significantly decreased the proliferation of SPCA1 lung cancer cells, whereas apoptosis was significantly increased $(\mathrm{P}<0.01)$ as determined by the MTS tetrazolium assay and flow cytometry analysis, respectively. At the molecular level, knockdown of RhoA resulted in the significant activation of caspase-3 $(\mathrm{P}<0.01)$, and a significant reduction in the levels of phosphorylated signal transducer and activator of transcription (phospho-STAT3; P<0.01), as determined by western blotting. The results suggested that RhoA knockdown prevents cell proliferation and induces apoptosis in SPCA1 lung cancer cells. Furthermore, the underlying mechanisms responsible for these effects may include the activation of caspase- 3 and the reduction of phospho-STAT3 levels.
\end{abstract}

\section{Introduction}

Medical interventions, including surgical resection, radiotherapy and chemotherapy, have provided therapeutic benefits for various lung cancer patients. However, tumor recurrence

Correspondence to: Dr Xueying Yang, Department of Thoracic Surgery, The Fourth Affiliated Hospital of China Medical University, 4 Chongshan East Road, Shenyang, Liaoning 110032, P.R. China

E-mail: 18904019778@163.com

Key words: Ras homolog family, member A, lung cancer, RNA interference, apoptosis, proliferation, molecular mechanisms may occur due to the development of multidrug resistance (1). Therefore, identification of the molecular pathways involved in the proliferation and apoptosis of lung cancer cells is of primary concern, as this may facilitate the development of inhibitors of these signaling pathways (2).

Cell migration, cell-to-cell adhesion, actomyosin contraction, mitosis and cytokinesis involve the Ras homolog (Rho)/Rho-kinase signaling pathway (3). In addition, numerous studies have demonstrated that the Rho/Rho-kinase pathway is prominently involved in cancer invasion, growth and metastasis, and Rho guanosine triphosphate hydrolases (GTPase) are involved in Ras-mediated oncogenic transformation $(4,5)$. Numerous cancers exhibit overexpression of members of the small GTPase Rho family, including Rho family members A, C, H, Ras-related C3 botulinum toxin substrate 1 and cell division control protein 42 (6-8). Therefore, the aim of the present study was to investigate whether the Rho/Rho-kinase signaling pathway may be involved in the proliferation and apoptosis of lung cancer cells $(9,10)$. A previous study demonstrated that the Rho-kinase inhibitor Fasudil, inhibited the Rho/Rho-kinase pathway, which subsequently inhibited the proliferation, invasion, adhesion and migration of lung cancer cells and induced apoptosis $(11,12)$.

The aim of the present study was to examine the role of RhoA in the proliferation and apoptosis of lung cancer cells. Loss of RhoA expression was achieved using RNA interference (RNAi) in SPCA1 lung carcinoma cells, and the consequences on cell proliferation and apoptosis were subsequently analyzed. The use of RNAi involves double-stranded RNA (dsRNA) sequences that are homologous to the target gene, which results in sequence-specific, post-transcriptional gene silencing (13). Stable SPCA1 cell lines with silenced RhoA expression were isolated following Geneticin (G418) screening. The authors then tested the hypothesis that RhoA affects lung cancer proliferation and apoptosis. A secondary aim was to identify the underlying molecular pathways involved in these processes.

\section{Materials and methods}

Cell culture. SPCA1 human lung carcinoma cells were purchased from the Cell Bank of Type Culture Collection of The Chinese Academy of Sciences (Shanghai, China). Cells were cultured in RPMI-1640 medium (Gibco; Thermo Fisher 
Scientific, Inc., Waltham, MA, USA) supplemented with $15 \%$ heat-inactivated fetal bovine serum (Thermo Fisher Scientific, Inc.), $100 \mathrm{mg} / \mathrm{ml}$ streptomycin and $100 \mathrm{U} / \mathrm{ml}$ penicillin (Beyotime Institute of Biotechnology, Haimen, China) at $37^{\circ} \mathrm{C}$ in a humidified atmosphere with $5 \% \mathrm{CO}_{2}$.

Vector synthesis. The authors used a BLAST program to design oligonucleotides based on a RhoA cDNA sequence in Genbank, which were produced by Takara Biotechnology Co., Ltd. (Dalian, China). The sequences were as follows: Small interfering (si) RhoA-B, '5-GCTAATACTAGCGGACTCC GATCTGCCGGAGTCCGCTAGTATTAGAAAAAACAGC TGTTCGA-5', and siRhoA-, 5'-GATCCGATTATGATCGCC TGAGGCTCAAGACGGCCTCAGGCGATCATAATCTTT TTGTCGACA-3'. The oligonucleotides and pRNATU6.1/Neo-SiRNA vector (Takara Biotechnology Co., Ltd.) were digested by BamHI and HindIII (New England BioLabs, Inc., Ipswich, MA, USA) overnight at $37^{\circ} \mathrm{C}$, then connected by T4 ligase (Thermo Fisher Scientific, Inc.) for $1 \mathrm{~h}$ at room temperature. Following this, the recombinant plasmid was transfected into DH5a (Takara Biotechnology Co., Ltd.) and screened overnight at $37^{\circ} \mathrm{C}$. The recombinant vector was selected from single colonies and amplified, finally confirmed by electrophoresis and DNA sequencing (14).

Determination of G418 screening concentration. SPCA1 cells during the logarithmic phase of growth were digested with $0.25 \%$ pancreatin (Hyclone; GE Healthcare Life Sciences, Logan, UT, USA) and seeded in 96-well plates. The following day, 11 different concentrations of G418 (Gibco; Thermo Fisher Scientific, Inc.; 0-1,000 $\mu \mathrm{g} / \mathrm{ml}$ in $100 \mu \mathrm{g} / \mathrm{ml}$ increments) were added to the wells. Six replicate wells were used for each concentration. In order to avoid the edge effect, the outer wells of the culture plate were not used. Cells were observed for 1 week, with the medium refreshed once in the middle of the week. Cells exhibited various rates of survival for concentrations of G418 between 0 and $400 \mu \mathrm{g} / \mathrm{ml}$, however a G418 concentration of between 500 and $1000 \mu \mathrm{g} / \mathrm{ml}$ resulted in comprehensive cell death by day 7. A concentration of $500 \mu \mathrm{g} / \mathrm{ml} \mathrm{G} 418$ was therefore selected as the screening concentration, and $200 \mu \mathrm{g} / \mathrm{ml} \mathrm{G} 418$ as the maintenance concentration.

RhoA siRNA transfection and establishment of stable cell lines. SPCA1 cells in the logarithmic phase of growth, and at passage number $<10$, were transfected with RhoA siRNA. Each transfection included a control group (transfected with empty vectors). SPCA1 cells ( $2 \times 10^{5}$ cells/well) were seeded in 6-well plates. Following incubation for $24 \mathrm{~h}$, cells were transfected with RhoA siRNA in serum-free medium using Lipofectamine ${ }^{\circledR} 2000$ (Invitrogen; Thermo Fisher Scientific, Inc.) in accordance with the manufacturer's protocol. A mixture containing $4 \mu \mathrm{g}$ vector and $10 \mu \mathrm{l}$ Lipofectamine $2000^{\mathrm{TM}}$ was incubated for $20 \mathrm{~min}$ at room temperature. SPCA1 cells were then washed and incubated with the vector-Lipofectamine mixture for $24 \mathrm{~h}$ in a $\mathrm{CO}_{2}$ incubator at $37^{\circ} \mathrm{C}$. A total of 500 and $200 \mu \mathrm{g} / \mathrm{ml} \mathrm{G} 418$ was then added to wells for three weeks in order to screen for and maintain the growth of stable RhoA siRNA-transfected cells, respectively. The recombinant plasmid RhoA-pRNAT-U6.1/Neo-SiRNA were selected from single colonies and amplified, so the SPCA1 cell lines expressing RhoA-pRNAT-U6.1/Neo-SiRNA could emit GFP continuously. Transfection was visualized using fluorescence microscopy (DFC365FX; Leica Microsystems GmbH, Wetzlar, Germany).

Western blot analysis. Stable RhoA siRNA-transfected cells and controls were subject to western blot analysis. To do this, cells were first washed three times with ice-cold PBS, before they were lysed in lysis buffer for $30 \mathrm{~min}$ in an ice-bath. Following electrophoresis (30 $\mu \mathrm{g} / \mathrm{well})$ on $10 \%$ SDS-PAGE gels, separated protein samples were transferred to a nitrocellulose membrane. The membrane was then blocked in 5\% non-fat dry milk in TBS with $0.05 \%$ Tween-20 (TBST) for $1 \mathrm{~h}$ at room temperature, to prevent nonspecific antibody binding. Following incubation with rabbit anti-RhoA antibody (dilution, 1:250; Jingmei Biotech Co., Ltd., Beijing, China) at $4^{\circ} \mathrm{C}$ overnight, the membrane was incubated with goat anti-rabbit antibody (dilution, 1:500; Jingmei Biotech Co., Ltd.). An antibody to $\beta$-actin (Sigma-Aldrich; Merck Millipore, Darmstadt, Germany) served as a loading control. For the detection of caspase-3 and signal transducer and activator of transcription (STAT) 3, cell lysates (40 $\mu \mathrm{g}$ each of protein) from stable RhoA siRNA-transfected and control cells were added to 2X Laemmli sample buffer (Hyclone; GE Healthcare Life Sciences), boiled for $5 \mathrm{~min}$ and electrophoresed under the aforementioned conditions with 10\% SDS-PAGE gels. Proteins were transferred to polyvinylidene difluoride membranes (Bio-Rad Laboratories, Inc., Hercules, CA, USA), rinsed with TBST ( $\mathrm{pH} 7.5$ ), and blocked in 5\% non-fat dry milk in TBST overnight at $4^{\circ} \mathrm{C}$. Membranes were incubated in polyclonal anti-STAT3, anti-caspase or anti-phosphorylated (phospho)-STAT3 (Tyr-705) primary antibodies for $3 \mathrm{~h}$. Following rinsing in TBST, the membrane was incubated with horseradish peroxidase-conjugated goat anti-rabbit antibody (EMD Millipore, Billerica, MA, USA) for $50 \mathrm{~min}$. Protein bands were detected by enhanced chemiluminescence (Pierce; Thermo Fisher Scientific, Inc.). Densitometry was used to semi-quantify band intensities.

Cell proliferation assay. An MTS tetrazolium assay was used to measure cell proliferation, as previously described (15). Briefly, RhoA siRNA-transfected cells or controls were seeded into 96-well plates (1,000 cells/well) in $100 \mu 1 /$ well of RPMI 1640 medium. Five replicates for each experimental group and time points $(0,24,48$ and $96 \mathrm{~h})$ were used. Following 24 h, $20 \mu \mathrm{l}$ CellTiter 96 AQueous One Solution (Promega Corporation, Madison, WI, USA) was added to each well, and cells were incubated at $37^{\circ} \mathrm{C}$ for a further $4 \mathrm{~h}$. Absorbance was measured at a wavelength of $490 \mathrm{~nm}$ each day for 7 days.

Fluorescence-activated cell sorting (FACS) analysis. Cell apoptosis in treated and untreated cells was measured by FACS or flow cytometry analysis, using the methods described previously (16). The FITC-Annexin V Apoptosis Detection kit (BD Pharmingen, San Diego, CA, USA) was used according to the manufacturer's protocol. Briefly, fluorescein isothiocyanate (FITC)-Annexin V and propidium iodide (PI) were used to stain siRNA RhoA-transfected and control cells for 5 min at room temperature. Annexin $\mathrm{V}^{-} \mathrm{PI}^{-}$(viable cells) and 
A

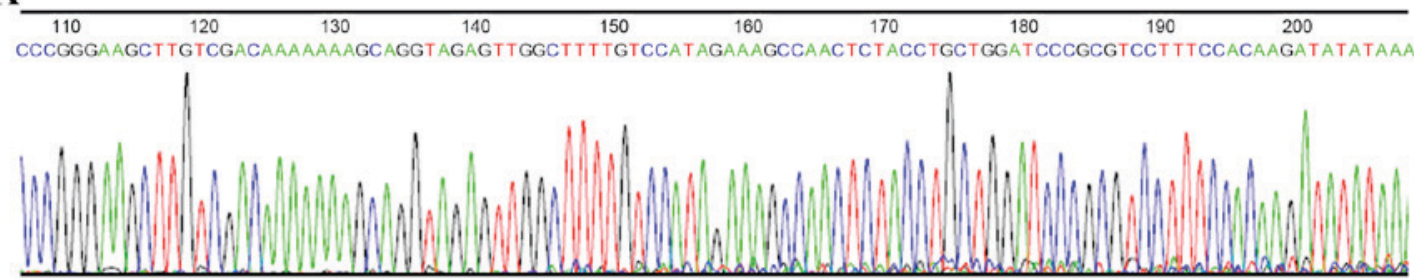

B

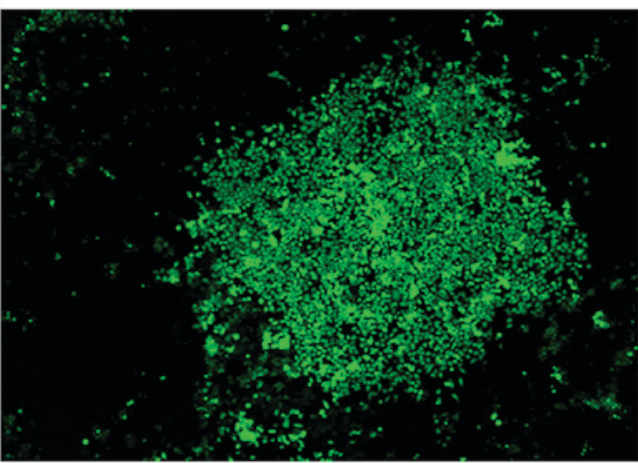

C

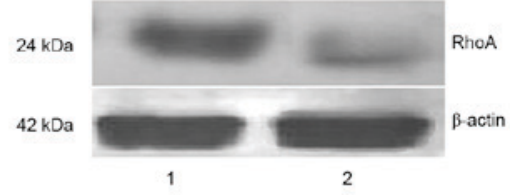

Figure 1. Generation of stable RhoA siRNA-transfected SPCA1 lung cancer cells and western blot analysis of RhoA protein expression. (A) Sequence analysis of recombinant plasmids and synthesized siRNA oligonucleotides. (B) RhoA siRNA-transfected SPCA1 cell lines under a fluorescence microscope (magnification, x50). (C) Western blot analysis of RhoA protein expression. Lane 1, control cells; lane 2, RhoA siRNA-transfected cells. Rho, Ras homolog family member A; siRNA, small interfering RNA.

Annexin $\mathrm{V}^{+}$(apoptotic) cells were evaluated by flow cytometry. A BD FACSCalibur flow cytometer (BD Biosciences, Franklin lakes, NJ, USA) was used to collect data that was then analyzed using BD CellQuest software (BD Biosciences).

Statistical analysis. Data are expressed as the mean \pm standard error. Variables were compared using the Student's t-test, the $\chi^{2}$ test and analysis of variance or Fisher's exact test. Data were analyzed using SPSS software (version 17.0; SPSS, Inc., Chicago, IL, USA). P<0.05 was considered to indicate a statistically significant difference.

\section{Results}

Successful generation of stable RhoA siRNA-transfected SPCA1 cell lines and western blot analysis of RhoA expression. In order to generate a RhoA-pRNAT-U6.1/Neo-SiRNA vector, oligonucleotide dsRNAs were ligated with pSUPER following annealing. The sequence of synthesized siRNA oligos and insert sequences were indistinguishable (Fig. 1A). Stable RhoA siRNA-transfected SPCA1 lung cancer cell lines were then generated (Fig. 1B). RhoA protein expression was analyzed by western blotting using an anti-RhoA antibody. As shown in Fig. 1C, a marked reduction in RhoA protein expression in RhoA siRNA-transfected cells was observed when compared with the control cells (Fig. 1C).

RhoA silencing decreases proliferation of cultured SPCAI lung cancer cells. The proliferation rate of stable RhoA siRNA-transfected cells was significantly lower when compared with the control cells every day for 7 days $(\mathrm{P}<0.01$; Fig. 2), as determined using the MTS assay. These results suggested that silencing of RhoA expression significantly decreased SPCA1 cell proliferation.

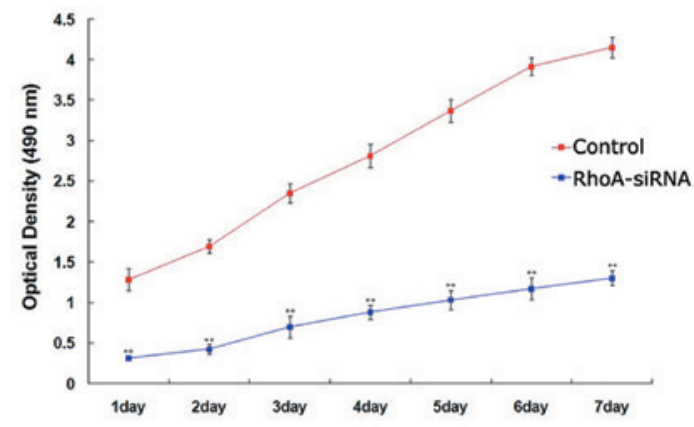

Figure 2. Effects of RhoA siRNA on the proliferation of cultured SPCA1 lung cancer cells. The proliferation of RhoA siRNA-transfected SPCA1 lung cancer cells was measured over the course of seven consecutive days using an MTS assay. Following RhoA knockdown, a significant decrease in the proliferation of SPCA1 cells was observed when compared with the control cells. ${ }^{* *} \mathrm{P}<0.01$ vs. control. RhoA, Ras homolog family member A; siRNA, small interfering RNA.

RhoA silencing increases apoptosis of cultured SPCA1 lung cancer cells. Flow cytometry was used to assess the level of apoptosis in RhoA siRNA-transfected SPCA1 cells. As shown in Fig. 3, stable RhoA siRNA-transfected cells exhibited a significant increase in the rate of apoptosis when compared with the control cells $(\mathrm{P}<0.01)$. These results suggested that RhoA silencing was associated with a marked increase in the level of apoptosis in SPCA1 cells.

RhoA silencing induces activation of caspase-3 in cultured SPCA1 lung cancer cells. As shown in Fig. 4, a significant increase in caspase- 3 expression in stable RhoA siRNA-transfected cells was observed when compared with the control cells, as determined by western blotting $(\mathrm{P}<0.01)$. By contrast, caspase- 9 activation remained unaffected between the two 

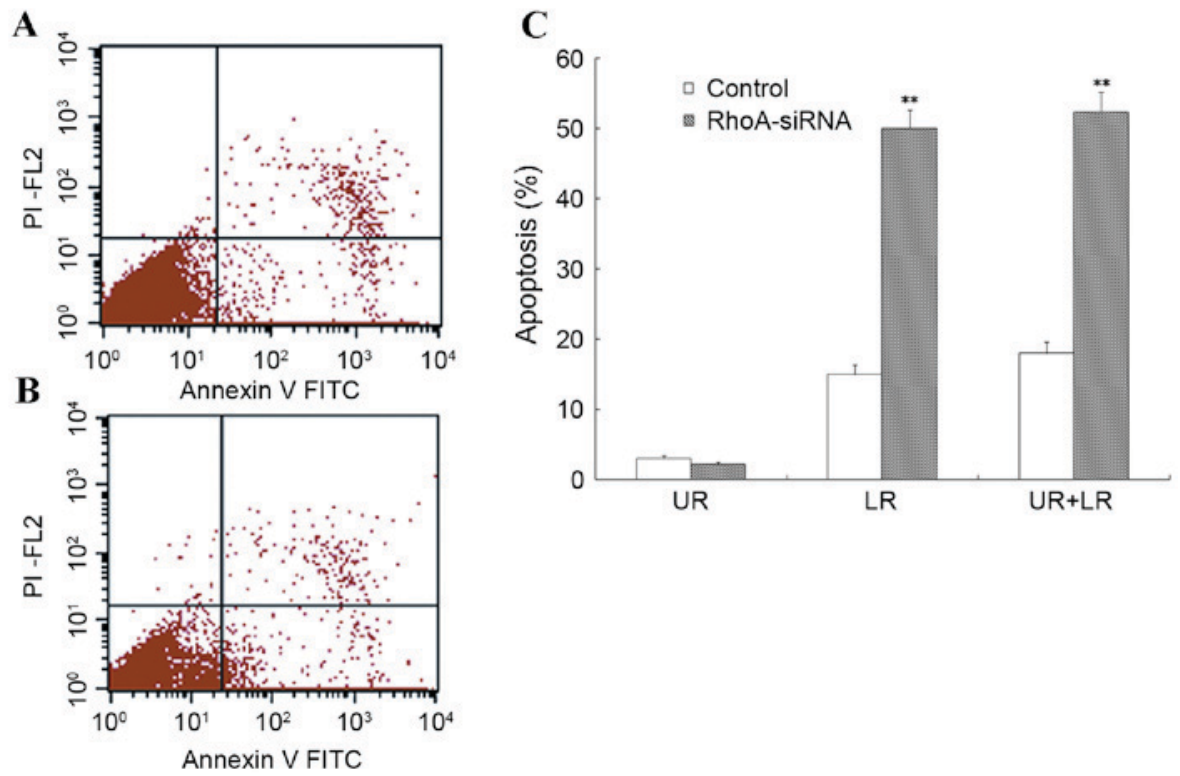

UR

A

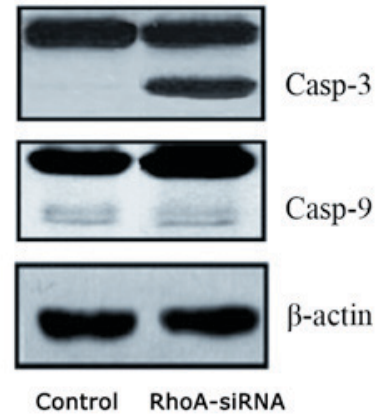

B

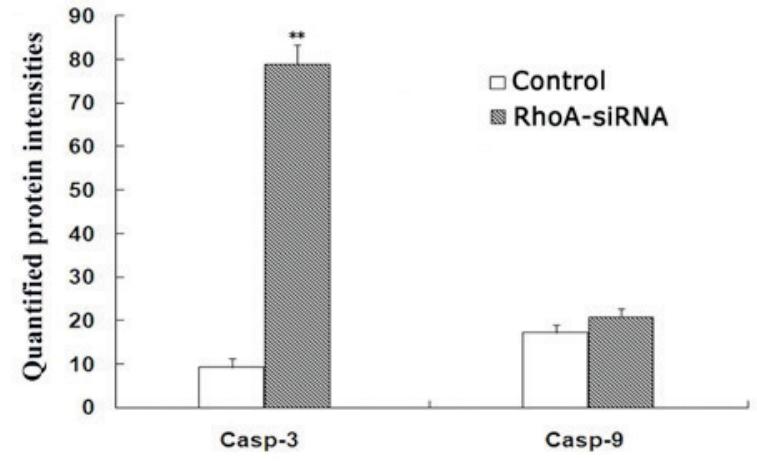

Figure 4. Activation of caspase-3 and caspase-9. (A) Western blot analysis of casp-3, casp- 9 and $\beta$-actin protein expression in RhoA siRNA-transfected SPCA1 cells and untreated controls using anti-casp-3, anti-casp- 9 and $\beta$-actin antibodies. $\beta$-actin was used as a loading control. (B) A significant increase in the active casp-3 form was observed in RhoA siRNA-transfected cells when compared with the control cells. By contrast, RhoA knockdown did not significantly alter casp-9 activation. ${ }^{* *} \mathrm{P}<0.01$ vs. control. RhoA, Ras homolog family member A; siRNA, small interfering RNA; casp-3, caspase-3; casp-9, caspase-9.

A

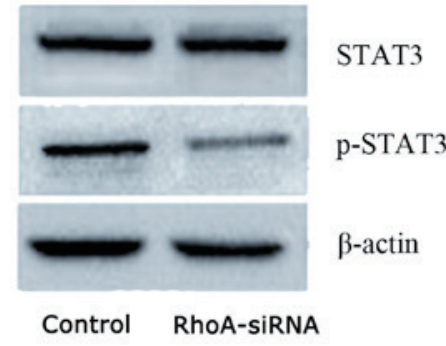

B

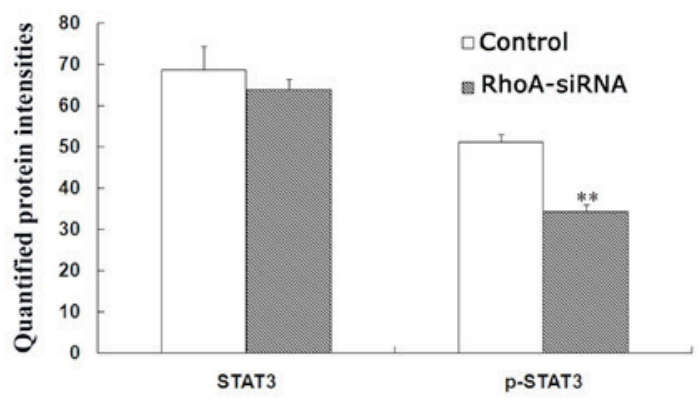

Figure 5. Protein expression levels of STAT3 and p-STAT3 in RhoA siRNA-transfected SPCA1 cells. (A) STAT3 and p-STAT3 (Tyr 705) protein expression levels were measured by western blotting and subsequent densitometry analysis (mean \pm standard error; $n=3$ ). (B) Quantification of STAT3 and p-STAT3 protein expression levels. Following RhoA knockdown, the level of p-STAT3 decreased by 31.5\% in RhoA siRNA-transfected cells, whereas RhoA siRNA did not significantly affect STAT3 protein levels. ${ }^{* *} \mathrm{P}<0.01$ vs. control. STAT3, signal transducer and activator of transcription 3; p-STAT3, phosphorylated-STAT3; RhoA, Ras homolog family member A; siRNA, small interfering RNA. 
groups (Fig. 4). These results suggest that RhoA silencing may activate caspase-3.

RhoA silencing downregulates STAT3 phosphorylation in cultured SPCA1 lung cancer cells. The level of phopho-STAT3 were decreased by $33.1 \%$ in stable RhoA siRNA-transfected cells when compared with control cells $(\mathrm{P}<0.01$; Fig. 5) as determined by western blotting analysis. By contrast, no significant difference in STAT3 protein expression levels between the groups was observed (Fig. 5). Therefore, RhoA silencing may decrease the expression levels of phospho-STAT3 in SPCA1 cells.

\section{Discussion}

The deregulation of cell proliferation and apoptosis pathways is a prerequisite for the development of lung cancer (17). At present, current cancer therapies consist of agents that target signaling pathways involved in cell proliferation, which result in cell death, for example, gemcitabine hydrochloride (18). However, further investigation into the mechanisms involved in cancer development is required in order to develop novel and improved anti-cancer therapies.

Activation of the Rho/Rho kinase pathway is important for cell migration, cell-to-cell adhesion, invasion and mitosis $(19,20)$, and may additionally be predominant in cancer growth and invasion (21). In non-small cell lung cancers, inhibition of the Rho/Rho-kinase pathway impedes tumour migration and invasion $(10,11)$. The aim of the present study was to assess the consequences of RhoA silencing in lung cancer cells at various stages of carcinogenesis, including proliferation and apoptosis.

RNAi is considered to be a precise method of gene silencing, which can involve the use of siRNA (22). This method was used to construct an RhoA siRNA expression vector in the present study, which was subsequently transfected into SPCA1 lung cancer cells. A stable RhoA siRNA-transfected SPCA1 cell line was successfully established and it was verified that RhoA siRNA reduced RhoA expression in SPCA1 lung cancer cells. It was demonstrated that knockdown of RhoA resulted in inhibition of lung cancer cell viability. Using flow cytometry analysis, it was subsequently demonstrated that a knockdown of RhoA significantly induced apoptosis in SPCA1 cells.

Western blot analysis was then performed in order to examine the activation of caspases as a possible mechanism underlying the increased levels of apoptosis observed in SPCA1 cells in response to RhoA knockdown. A significant increase in caspase-3, but not caspase-9 expression, was observed in RhoA siRNA-transfected SPCA1 cells. This was consistent with the results obtained from FACS analysis. Therefore, RhoA knockdown induced strong activation of caspase-3 in SPCA1 cells, which suggests that caspase-dependent apoptosis may have contributed to the RhoA knockdown-mediated, anti-proliferative effects observed in SPCA1 cells.

Cellular transformation in response to constitutive activation of STAT3 is frequently observed in numerous human cancer cells (23). In the present study, western blotting was used to determine whether the observed RhoA siRNA-mediated inhibition of SPCA1 cell proliferation was the result of STAT3 inactivation, by determining the phosphorylation status of STAT3. RhoA knockdown resulted in a significant decrease in phospho-STAT3 (Tyr-705) levels, however, no effect on total STAT3 protein levels was observed. The results suggested that RhoA knockdown induced cell growth inhibition and apoptotic cell death in SPCA1 cells, which may be associated with STAT3 inactivation.

In conclusion, the results of the present study suggest that RhoA may be important for lung cancer cell proliferation and apoptosis. Alterations in caspase-3 and phospho-STAT3 levels and activation may be the underlying molecular mechanisms associated with the effect of RhoA on SPCA1 cell proliferation and apoptosis. These results provide an improved understanding of the cellular pathways involved in human lung cancer, and may facilitate the development of novel therapies to treat patients with this disease.

\section{Acknowledgements}

The present study was supported by The Specialized Research Fund for the Doctoral Program of Higher Education of China (grant no. 20122104120015).

\section{References}

1. Lee C, Raffaghello L and Longo VD: Starvation, detoxification, and multidrug resistance in cancer therapy. Drug Resist Updat 15: 114-122, 2012.

2. Street CA and Bryan BA: Rho kinase proteins-pleiotropic modulators of cell survival and apoptosis. Anticancer Res 31: 3645-3657, 2011

3. Somlyo AP and Somlyo AV: $\mathrm{Ca}^{2+}$ sensitivity of smooth muscle and nonmuscle myosin II: Modulated by $\mathrm{G}$ proteins, kinases, and myosin phosphatase. Physiol Rev 83: 1325-1358, 2003.

4. Zicha D, Dobbie IM, Holt MR, Monypenny J, Soong DY, Gray C and Dunn GA: Rapid actin transport during cell protrusion. Science 300: 142-145, 2003.

5. Brown M, Roulson JA, Hart CA, Tawadros T and Clarke NW: Arachidonic acid induction of Rho-mediated transendothelial migration in prostate cancer. Br J Cancer 110: 2099-2108, 2014.

6. Ridley AJ: Rho proteins and cancer. Breast Cancer Res Treat 84: 13-19, 2004.

7. Sahai E and Marshall CJ: RHO-GTPases and cancer. Nat Rev Cancer 2: 133-142, 2002.

8. Burbelo P, Wellstein A and Pestell RG: Altered Rho GTPase signaling pathways in breast cancer cells. Breast Cancer Res Treat 84: 43-48, 2004

9. Morgan-Fisher M, Wewer UM and Yoneda A: Regulation of ROCK Activity in cancer. J Histochem Cytochem 61: 185-198, 2013.

10. Amano M, Nakayama M and Kaibuchi K: Rho-kinase/ROCK: A key regulator of the cytoskeleton and cell polarity. Cytoskeleton (Hoboken) 67: 545-554, 2010.

11. Yang X, Liu Y, Zong Z and Tian D: The Rho kinase inhibitor fasudil inhibits the migratory behaviour of 95-D lung carcinoma cells. Biomed Pharmacother 64: 58-62, 2010.

12. Yang X, Di J, Zhang Y, Zhang S, Lu J, Liu J and Shi W: The Rho-kinase inhibitor inhibits proliferation and metastasis of small cell lung cancer. Biomed Pharmacother 66: 221-227, 2012.

13. Bass BL: Double-stranded RNA as a template for gene silencing. Cell 101: 235-238, 2000.

14. Yang X, Zheng F, Zhang S and Lu J: Loss of RhoA expression prevents proliferation and metastasis of SPCA1 lung cancer cells in vitro. Biomed Pharmacother 69: 361-366, 2015.

15. Shin JY, Kim JO, Lee SK, Chae HS and Kang JH: LY294002 may overcome 5-FU resistance via down-regulation of activated p-AKT in Epstein-Barr virus-positive gastric cancer cells. BMC Cancer 10: 425, 2010.

16. Chen T, Xu Y, Guo H, Liu Y, Hu P, Yang X, Li X, Ge S, Velu SE, Nadkarni DH, et al: Experimental therapy of ovarian cancer with synthetic makaluvamine analog: In vitro and in vivo anticancer activity and molecular mechanisms of action. PLoS One 6: e20729, 2011. 
17. Morgensztern D, Campo MJ, Dahlberg SE, Doebele RC, Garon E, Gerber DE, Goldberg SB, Hammerman PS, Heist RS, Hensing T, et al: Molecularly targeted therapies in non-small cell lung cancer annual update 2014. J Thorac Oncol 10 (1 Supp 1) S1-S63, 2015

18. Pooja D, Panyaram S, Kulhari H, Reddy B, Rachamalla SS and Sistla R: Natural polysaccharide functionalized gold nanoparticles as biocompatible drug delivery carrier. Int J Biol Macromol 80: 48-56, 2015.

19. Ying H, Biroc SL, Li WW, Alicke B, Xuan JA, Pagila R, Ohashi Y, Okada T, Kamata Y and Dinter H: The Rho kinase inhibitor fasudil inhibits tumor progression in human and rat tumor models. Mol Cancer Ther 5: 2158-2164, 2006.

20. Rath $\mathrm{N}$ and Olson MF: Rho-associated kinases in tumorigenesis: Re-considering ROCK inhibition for cancer therapy. EMBO Rep 13: 900-908, 2012.
21. Nakajima M, Hayashi K, Katayama K, Amano Y, Egi Y, Uehata M, Goto N and Kondo T: Wf-536 prevents tumor metastasis by inhibiting both tumor motility and angiogenic actions. Eur J Pharmacol 459: 113-120, 2003.

22. Wagner A, Röhrs V, Kedzierski R, Fechner H and Kurreck J: A novel method for the quantification of adeno-associated virus vectors for RNA interference applications using quantitative polymerase chain reaction and purified genomic adeno-associated virus DNA as a standard. Hum Gene Ther Methods 24: 355-363, 2013.

23. Yu H and Jove R: The STATs of cancer-new molecular targets come of age. Nat Rev Cancer 4: 97-105, 2004. 